\title{
Oncogenic BRAF mutations and p16 expression in melanocytic nevi and melanoma in the Polish population
}

\author{
Małgorzata Mackiewicz-Wysocka1, Patrycja Czerwińska'2,3, Violetta Filas ${ }^{4,5}$, Elżbieta Bogajewska ${ }^{5}$, Agata Kubicka \\ Anna Przybyła ${ }^{2}$, Ewelina Dondajewska², Tomasz Kolenda ${ }^{2,3,6}$, Andrzej Marszałek ${ }^{4,5}$, Andrzej Mackiewicz ${ }^{2,3}$ \\ ${ }^{1}$ Chair and Department of Dermatology, Poznan University of Medical Sciences, University Hospital No. 2, Poznan, Poland \\ ${ }^{2}$ Chair of Biotechnology, Department of Cancer Immunology, Poznan University of Medical Sciences, Medical Biology Centre, Poznan, \\ Poland \\ ${ }^{3}$ Department of Cancer Diagnostics and Immunology, Greater Poland Cancer Centre, Poznan, Poland \\ ${ }^{4}$ Department of Clinical Pathology, Poznan University of Medical Sciences, Poznan, Poland \\ ${ }^{5}$ Department of Cancer Pathology, Greater Poland Cancer Centre, Poznan, Poland \\ ${ }^{6}$ Postgraduate School of Molecular Medicine, Medical University of Warsaw, Warsaw, Poland
}

Adv Dermatol Allergol 2017; XXXIV (5): 490-498 DOI: https://doi.org/10.5114/ada.2017.71119

\begin{abstract}
Introduction: Twenty-five - fifty percent of skin melanomas arise from nevi. Melanocyte proliferation is activated by $B R A F^{\mathrm{V} 600 \mathrm{E}}$, then is arrested, but single nevi transform to melanomas. p16 controls arrest, and p16 loss may promote transformation.

Aim: To analyze $B R A F^{\mathrm{V} 600 \mathrm{E}}$, p16 expression and melanocyte proliferation in dermal, compound and dysplastic nevi, cells of primary and metastatic melanoma in the Polish population.

Material and methods: One hundred and thirty-two nevi (dermal, compound, dysplastic) and 41 melanomas (in situ, primary, metastatic) were studied. BRAF was assessed by cobas ${ }^{\circledR} 4800$ BRAF ${ }^{600}$ Mutation Test, High Resolution Melting Assay validated with: pyrosequencing and immunohistochemistry. p16 and Ki67 expression was analyzed by IHC.

Results: Eighty-two percent of nevi and $57 \%$ of melanomas display $B R A F^{\mathrm{V} 600 \mathrm{E}}$ expression. Most dermal and compound nevi had $>50 \%$ of p16(+) cells. BRAF ${ }^{\mathrm{V} 600 \mathrm{E}}$ dysplastic nevi had a low number of p16(+) cells. Nevi without $B R A F^{V 600 E}(\mathrm{WT})$, had $90 \%$ of cells p16(+). In $60 \%$ of in situ and primary melanomas, there was a low number of cells of p16(+). Fifty percent of WT metastatic melanoma and 33\% of BRAF ${ }^{\mathrm{V} 600 \mathrm{E}}$ showed a high level of p16. The number of $\mathrm{Ki} 67(+)$ cells in dysplastic nevi was very low. In $25 \%$ of $B R A F^{\mathrm{V} 600 \mathrm{E}}$ melanomas in situ and $55 \%$ of WT, $>10 \%$ cells were $\mathrm{Ki} 67(+)$. All BRAF $F^{\mathrm{V} 600 \mathrm{E}}$ primary melanomas and $66 \%$ of WT had $>10 \% \mathrm{Ki} 67(+)$ cells. Twenty percent of $B R A F^{\mathrm{V} 600 \mathrm{E}}$ and WT metastases had $>10 \%$ of Ki67(+), however, $62 \%$ of $B R A F^{\mathrm{V} 600 \mathrm{E}}$ and $32 \%$ of WT samples had $>50 \%$ of Ki67(+) cells. Conclusions: $B R A F^{\mathrm{V} 600 \mathrm{E}}$ and $\mathrm{p} 16$ are more frequent in nevi than in melanoma in vivo. A significantly higher p16 expression was observed in mutated nevi than in WT, while in melanoma it was just the opposite. The proliferation rate of melanoma cells negatively correlated with p16 expression.
\end{abstract}

Key words: melanoma, nevi, BRAF, CDKN2A, p16, Ki67.

\section{Introduction}

Melanoma is a very heterogeneous disease. It shows a significant diversity in presentation, incidence and mortality in various countries, ethnic groups or patients with socioeconomic status [1]. The diversity is in part related to both histopathological [1] and genomic [2] melanoma subtypes, germline genetic factors predisposing or affecting melanoma development and progression [3] or environmental factors such as ultraviolet (UV) exposure [4]. Approximately $25-50 \%$ of skin melanomas arise or are associated with pre-existing benign melanocytic nevi [5]. On the other hand, nevi rarely transform to melanoma either exposed or not exposed to UV [6]. Recently, significant progress in understanding of the nevi progression to melanoma has been made in vitro, in animal models or human in vivo clinical studies [7, 8].

BRAF is serine/threonine protein kinase B-raf, constituent of the ERK1/2-signaling pathway involved in regulation of cells proliferation, differentiation and survival [9]. Active mutations in BRAF most frequent V600E

Address for correspondence: Małgorzata Mackiewicz-Wysocka MD, PhD, Chair and Department of Dermatology, Poznan University of Medical Sciences, University Hospital No 2, 39 Przybyszewskiego St, 60-355 Poznan, Poland, phone: +48 618691285 , fax: +48 6186915 72, e-mail: mmackwys@ump.edu.pl Received: 11.10.2017, accepted: 17.10.2017. 
$\left(B R A F^{\mathrm{V} 600 \mathrm{E}}\right)$ are driver mutations in melanoma pathogenesis [9]. Moreover, a high frequency of $B R A F^{\mathrm{V} 600 \mathrm{E}}$ is observed in nevi [10], which mostly develop during the first 25 years of life. Paradoxically, BRAF ${ }^{\mathrm{V} 600 \mathrm{E}}$ also induces p16 that in response halts proliferating melanocytes [11]. Nevi melanocytes proliferation is transiently activated by $B R A F^{\mathrm{V} 600 \mathrm{E}}$. It is usually stopped when nevi reach $3-5 \mathrm{~mm}$ in diameter. Then nevi melanocytes proliferation is arrested or some nevi regress in the advanced age despite the expression of the BRAF $F^{\mathrm{V} 600 \mathrm{E}}$ for decades [11]. However, the arrest is reversible since a small fraction of nevi transforms to skin melanomas [12]. Originally it was proposed that nevi are senescent tumors [10]. However, later studies analyzing expression of effectors of senescence including p16 ${ }^{\text {INK4a }}$, p53 and DNA damage $(\gamma-\mathrm{H} 2 \mathrm{AX})$ and predictive markers of senescence such as Ki67, PML, senescence-associated $\beta$-galactosidase shown that nevi cells could not be distinguished from their precursors (skin melanocytes) and transformed melanocytes (melanoma cells). Accordingly, the concept that human nevi are arrested by senescence was challenged [13].

Oncogene-induced arrest is promoted by a high expression of cyclin-dependent (CDK) kinase inhibitors CDKN2A encoded p16 and CDKN2B encoded p15 [8]. p15 and p16 were highly expressed in isolated nevus melanocytes, while in isolated epidermal melanocytes from adjacent tissue displayed a low expression. In experimental models, primary melanocytes transduced with p15 halted proliferation, while transduced with p16 continued to proliferate but more slowly than controls [8]. In melanoma, both p15 and p16 expression was decreased [14]. Deletion of chromosome arm 9p, which contains the CDKN2A and CDKN2B genes, is common in primary melanoma [15]. In $90 \%$ of deletions of CDKN2A gene, the deletion of adjacent CDKN2B gene is also observed [16].

Mutations or polymorphic variants of CDKN2A are linked with predisposition to sporadic and hereditary melanoma $[17,18]$. Variation in the phenotype and the nevi number within population is mostly genetically determined, for example A148T variant showed a protective role on the number of atypical nevi in the family sample [17]. In the Polish population, germ-line mutations are rare though there are three common polymorphic variants of CDKN2A, A148T, Nt500c $>g$ and Nt540c $>$ t. Variant A148T was shown to be associated with an increased risk of melanoma [19-21].

\section{Aim}

The aim of the study was to analyze oncogenic $B R A F^{\mathrm{V} 600 \mathrm{E}}$ and $\mathrm{p} 16$ expression as well as melanocyte proliferation in dermal, compound and dysplastic nevi, and melanoma cells of primary and metastatic melanoma in the Polish population.

\section{Material and methods}

\section{Patient samples}

The study was carried out at the Poznan Medical University (PMU) and Greater Poland Cancer Centre (GPCC). It was a retrospective study approved by the Bioethics Committee of PMU. Melanoma and nevi formalin-fixed, paraffin-embedded tissue blocks (FFPE) were obtained from archives of the Department of Dermatology, PUM and Department of Cancer Pathology, GPCC, Poznan, Poland. Samples were collected from patients in 20132016. There were FFPE obtained from 173 subjects (41 melanoma patients, 132 healthy individuals with nevi), which were randomly selected for studies and evaluated by two independent investigators (pathologists, E.B. and A.Mar.). Nevi were diagnosed as dermal (89 samples), compound (30) and dysplastic (13). Melanoma samples were described as melanoma in situ (20 samples), primary melanoma Clark III/IV (7) and metastatic (14; lymph node metastases -10 , other metastases -4 samples).

\section{DNA isolation}

For genomic DNA isolation, the $\operatorname{COBAS}^{\circledR}$ DNA Sample Preparation Kit (Roche) was used according to the protocol provided by the manufacturer. Briefly, one $5 \mu \mathrm{m}$ thick section from FFPE was deparaffinized and then lysed in DNA Tissue Lysis Buffer supplemented with Proteinase K. DNA Binding Buffer and isopropanol were added to the samples and the lysates were placed on a High Pure Filter Tube. Samples were washed twice and the DNA was eluted with DNA Elution Buffer. The concentration was measured with NanoDrop ${ }^{\mathrm{TM}} 2000$ Spectrophotometer (Thermo Fisher). DNA was stored in $-80^{\circ} \mathrm{C}$.

\section{Determination of BRAF status}

Two different methods were utilized for BRAF status determination: (i) cobas $^{\oplus} 4800$ BRAF V600 Mutation Test and (ii) High Resolution Melting (HRM) Assay [22], which was further validated with two tests: pyrosequencing of BRAF exon 15 and immunohistochemistry (IHC) with antiBRAF V600E (VE1) Mouse Monoclonal Primary Antibody (Ventana). Out of 173 FFPE samples, 126 were tested with cobas $^{\oplus} 4800$ BRAF V600 Mutation Test according to the protocol provided by the manufacturer. Forty-eight samples (both melanoma and nevi) were analyzed with HRM method further validated by both sequencing and IHC. BRAF ${ }^{\mathrm{V} 600 \mathrm{E}}$ mutation was considered present when confirmed by at least two tests [23].

\section{Immunohistochemistry}

From nevi and melanoma, FFPET sections of $4-5 \mu \mathrm{m}$ were cut, mounted on the adhesive slides and subjected to IHC using CINtec p16 Histology (E6H4 clone) to detect p16 ${ }^{\text {INK4a }}$. The IHC used RoView's ultraView Universal DAB Detection Kit. Immunoperoxidase staining was 
performed on BenchMark Ultra from Ventana Medical System (Roche Diagnostics). As an external p16 positive control, an earlier validated squamous carcinoma specimen was used. For semiquantitative assessment of p16, 12-point score was calculated. It was based on the 4-point scale of staining intensity $(0$ - indicates no staining, 1 - weak positive, 2 - positive, 3 - highly positive) and on a 4-point percentage scale of the number of stained cells ( $1-10 \%$ and below of stained cells, $2-$ $11-50 \%, 3-51-75 \%$ of stained cells and $4-76-100 \%)$. The final score was obtained by multiplying the degree of intensity by percentage of stained cells. Two independent investigators scored the samples (V.F. and A.Mar).

For Ki67, monoclonal antibody RTU Clone MIB-1 and IHC kit En Vision ${ }^{\text {TM }}$ FLEX GV800 (DAKO, Denmark) were used. The IHC staining was carried out using automated system OMNIS (DAKO). As positive control for Ki67 tonsi tissue was applied. The result obtained was expressed as a number (in percent) of stained cells: $1-10 \%, 11-50 \%$, $51-75 \%$ and $76-100 \%$.

\section{Statistical analysis}

All statistical analyses were performed with GraphPad Prism 6. Continuous variables are given as means \pm SD unless otherwise specified. Chi-squared test was used for comparisons of proportions. We used Mann Whitney test and Kruskal-Wallis test to compare means of two or more than two groups, respectively. Spearman correlation was used to analyze the p16 and Ki67 expression. All given $p$-values are two-tailed and a $p$-value $<0.05$ was regarded statistically significant.

\section{Results}

\section{Expression of $B R A F^{\mathrm{V} 600 \mathrm{E}}$}

Expression of $B R A F^{\mathrm{V} 600 \mathrm{E}}$ was found in 108 out of 132 nevi (82\%) and in 24 out of 41 melanomas studied (58\%) (Figure $1 \mathrm{~A}$ ). The frequency of BRAF ${ }^{\mathrm{V} 600 \mathrm{E}}$ mutation in our melanoma samples corresponds to the previously reported high level of BRAF ${ }^{\mathrm{V} 600 \mathrm{E}}$ mutation in TCGA data (163 samples out of 366 sequenced melanomas (44.5\%) display BRAF ${ }^{\mathrm{V} 600 \mathrm{E}}$ mutation according to www.cbioportal. org) $[16,24]$.

Eighty-two out of 89 dermal nevi (92\%), 21 out of 30 compound nevi (70\%), 5 out of 13 dysplastic nevi (38\%), 12 out of 21 melanomas in situ (57\%), 4 out of 7 primary melanomas Clark III/IV (57\%) and 8 out of 14 metastatic melanomas (57\%) were BRAF ${ }^{\mathrm{V} 600 \mathrm{E}}$ positive. There was a statistically significant difference in $B R A F^{\mathrm{V} 600 \mathrm{E}}$ frequency between nevi and melanoma ( $p<0.0001$ ) (Figure $1 \mathrm{~B}$ ). Intensity of positive anti-BRAF ${ }^{\mathrm{V} 600 \mathrm{E}}$ staining in IHC was significantly higher in melanomas than in nevi (Figure 2).

\section{Expression of $\mathrm{p} 16$}

Expression of p16 was observed in all but 3 cases, which included one compound nevus, one dysplastic nevus and one melanoma case. In dermal and compound nevi, there are $90 \%$ and $80 \%$ of samples with more than $50 \%$ of p16 positive cells, respectively (Figure $3 \mathrm{~A}$ ). In compound nevi with BRAF mutation, more than half of cases had a significantly lower percentage of 16 positive cells (below 50\%) as opposed to compound nevi without BRAF mutation (referred to as wild type - WT), which
A

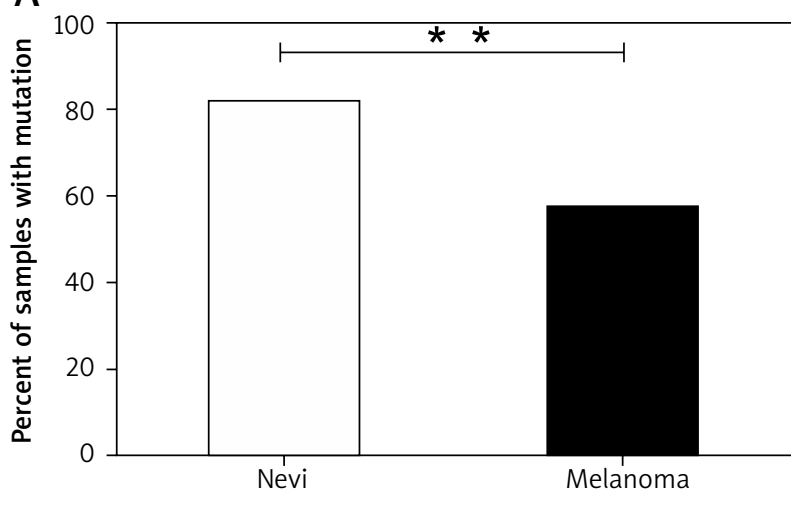

B

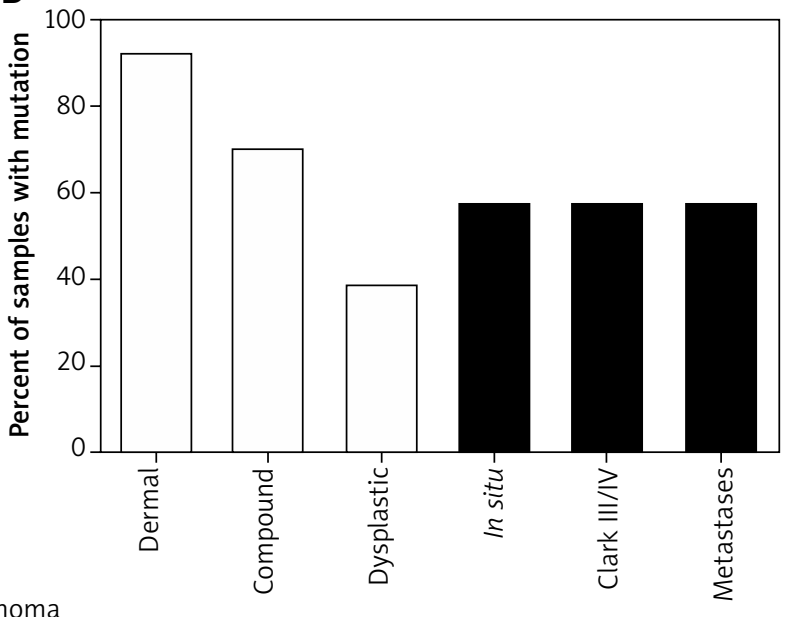

Figure 1. The frequency of BRAF ${ }^{\mathrm{V} 000 \mathrm{E}}$ point mutation in nevi and melanoma samples. A - The BRAF ${ }^{\mathrm{G} 00 \mathrm{E}}$ mutation was detected in 108 out of 132 nevi samples (82\%) and 24 out of 42 melanoma samples (57\%). B - Dermal nevi are characterized by the highest frequency of BRAF ${ }^{\mathrm{V} 600 \mathrm{E}}$ mutation (92\%) when compared to compound or dysplastic nevi. The frequency of $\mathrm{BRAF}^{\mathrm{V} 600 \mathrm{E}}$ mutation among melanoma samples is similar. Error bars $=\mathrm{SD},{ }^{* *} p<0.01$ 


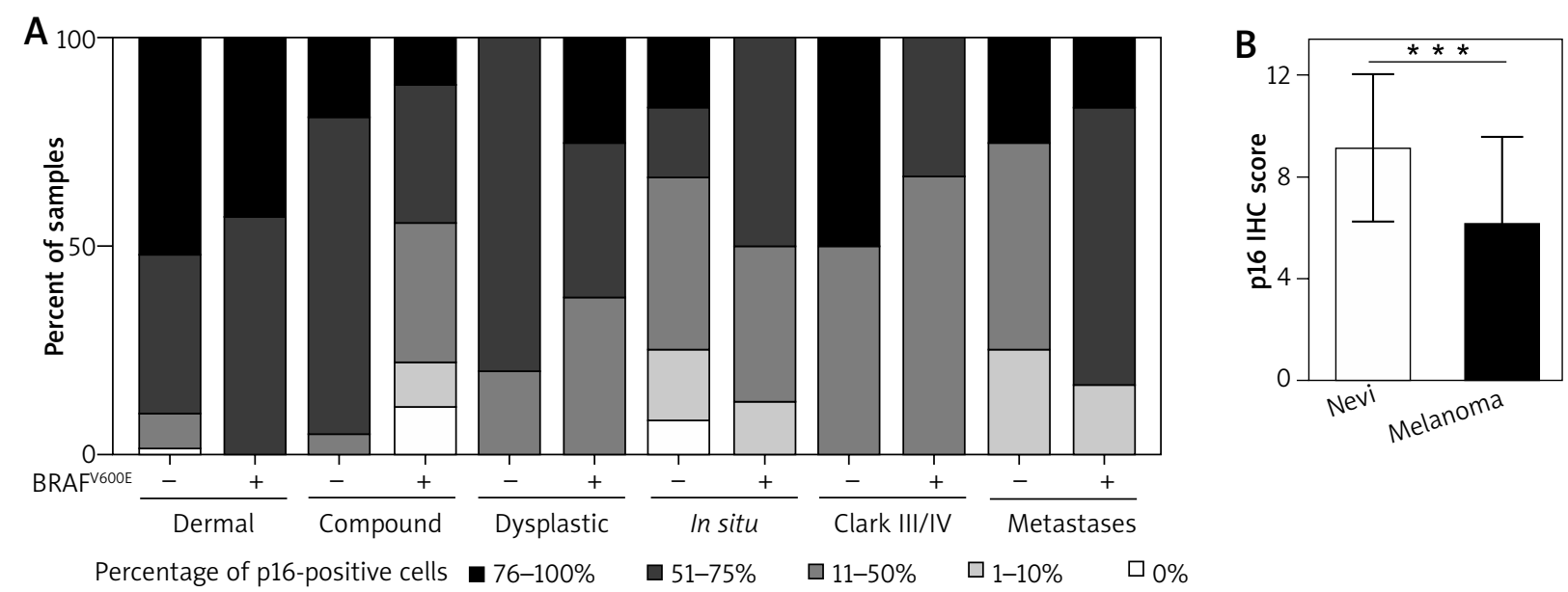

C

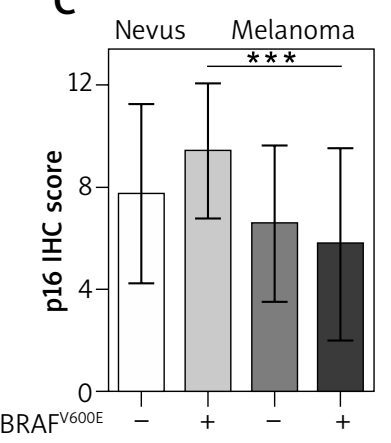

D
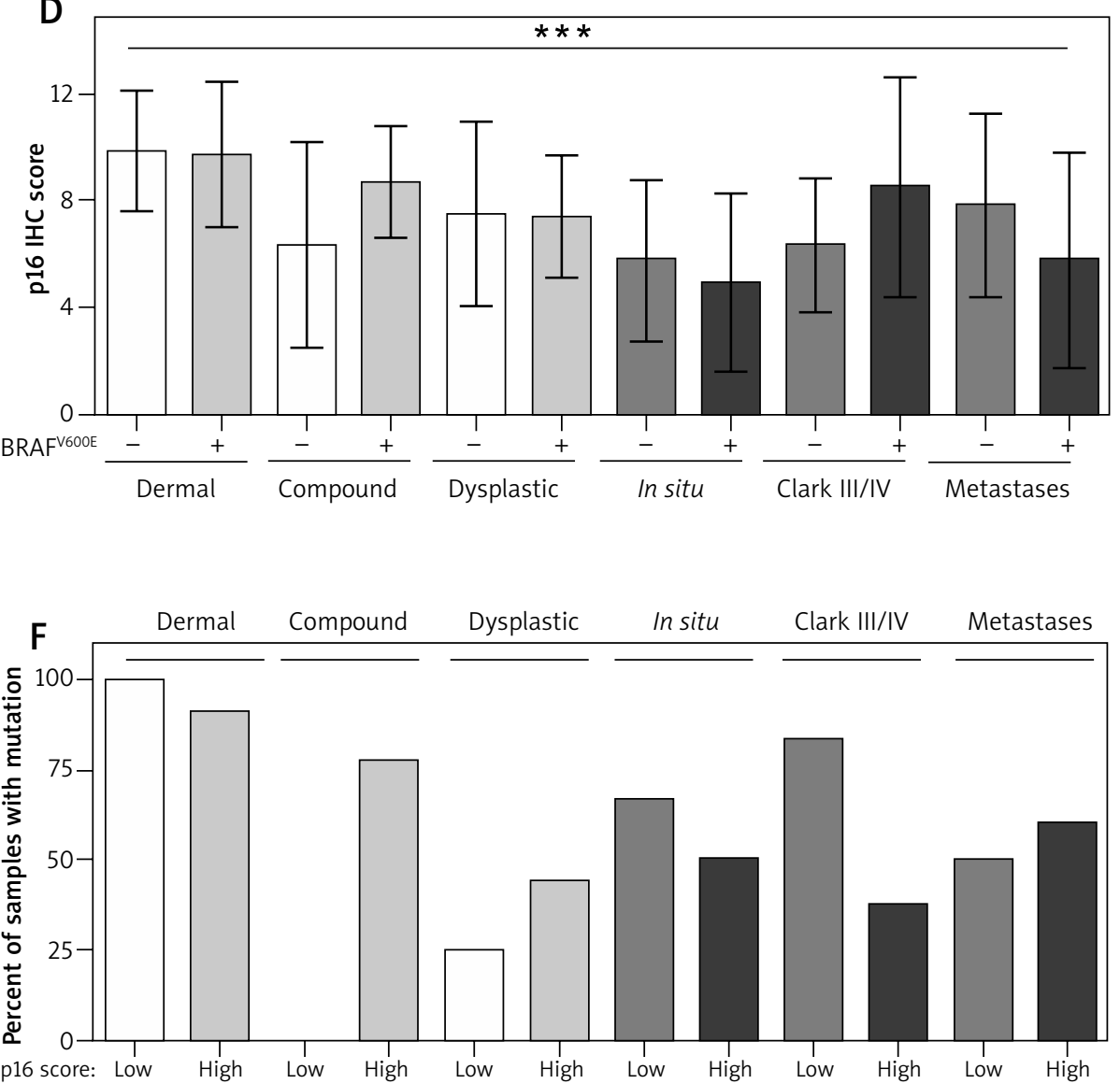

p16 score: Low High Low High Low High Low High Low High Low High
$\mathrm{E}$

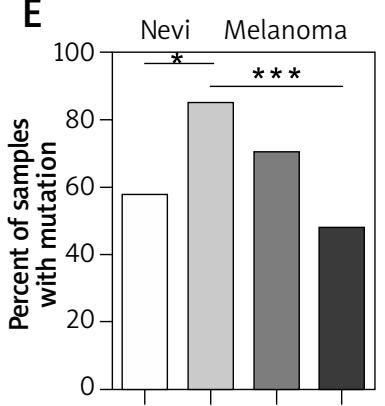

p16 score: Low High Low High

Figure 2. The p16 immunohistochemistry score differs significantly between nevi and melanoma samples. A - The frequency of p16-positive cells in nevi and melanoma samples according to the pathological classification and the BRAF mutation status. 5 -point percentage scale of the number of positive cells was used $(0=$ no positive cells, $1=1-10 \%$ of positive cells, $2=11-50 \%, 3=51-75 \%$ and $4=76-100 \%)$. B - The average p16 IHC score is significantly higher in nevi when compared to melanoma samples $(p<0.0001)$. C - The average p16 IHC score in nevi and melanoma according to the BRAF mutation status. D - The average p16 IHC score in nevi and melanoma samples according to the pathological classification. Melanoma in situ is characterized by the lowest level of p16 IHC score. $\mathrm{E}-$ The frequency of BRAF ${ }^{\mathrm{V} 600 \mathrm{E}}$ mutation in nevi and melanoma samples according to the level of p16 IHC score (low score $\leq 6$, high score $>6$ ). BRAF ${ }^{\mathrm{V} 600 \mathrm{E}}$ mutation is more frequent in nevi with high p16 $(p<0.05)$ in comparison to low p16 nevi; in contrast, BRAF ${ }^{6600}$ mutation is slightly less frequent in melanoma with high p16 when compared to low p16 samples. F - The frequency of BRAF ${ }^{\mathrm{V} 600 \mathrm{E}}$ mutation in nevi and melanoma samples according to the level of p16 IHC score (low score $\leq 6$, high score $>6$ ) and the pathological classification. Error bars $=\mathrm{SD},{ }^{* * *} p<0.0001$ 
have shown more than $50 \%$ of p16 expressing cells in $90 \%$ of cases (Figure $3 \mathrm{~A}$ ). In melanoma in situ and primary melanoma (Clark III/IV), about $60 \%$ of cases showed a low (below 50\%) number of cells expressing p16. In metastatic melanoma WT about 50\%, while in BRAF $F^{\mathrm{V} 600 \mathrm{E}}$ only $33 \%$, of cases showed a high p16 expression (above $50 \%$ cells positive) (Figure $3 \mathrm{~A}$ ).

In nevi, the p16 expression (average IHC score $=9.129$ $\pm 2.883)$ was statistically higher $(p<0.0001)$ than in melanoma (average IHC score $=6.122 \pm 3.480$ ) (Figure 3 B) In WT nevi, p16 expression (average IHC score $=7.750$ \pm 3.505 ) was lower than in BRAF ${ }^{\mathrm{V} 600 \mathrm{E}}$ nevi (average IHC score $=9.435 \pm 2.684$ ), while in WT melanoma it was higher (average $\mathrm{IHC}$ score $=6.588 \pm 3.063$ ) than in $B R A F^{\mathrm{F} 600 \mathrm{E}}$ melanoma (average IHC score $=5.792 \pm 3.776$ ), although with no statistical significance (Figure $3 \mathrm{C}$ ). However, in mutated $B R A F^{\mathrm{V} 600 \mathrm{E}}$ nevi, p16 expression was significantly higher than in BRAF ${ }^{\mathrm{V} 600 \mathrm{E}}$ melanoma $(p<0.0001)$. In WT and $B R A F^{\mathrm{V} 600 \mathrm{E}}$ dermal nevi, p16 expression was at the same level (average IHC score in WT $=9.744 \pm 2.725$ and in $B R A F^{\mathrm{V} 600 \mathrm{E}}=9.857 \pm 2.268$ ), however it slightly differs in compound nevi (average IHC score in WT $=8.714 \pm 2.101$ and in $\left.B R A F^{\mathrm{V} 600 \mathrm{E}}=6.333 \pm 3.841\right)$ but with no statistical significance (Figure $3 \mathrm{D})$.

Due to variations in p16 expression within groups studied we decided to divide patients in two subgroups, one with high and the other with low p16 expression (Low and High p16 IHC score with cutoff expression of 6, respectively) and evaluate the frequency of $B R A F^{\mathrm{V} 600 \mathrm{E}}$ in nevi and melanoma separately (Figure $3 \mathrm{E}$ ). In nevi with low p16 and nevi with high p16, there were $57 \%$ and $85 \%$ of $B R A F^{\mathrm{V} 600 \mathrm{E}}$ carriers, respectively $(p<0.01)$. On the other hand, in melanoma with low p16, there were $70 \%$ of BRAF $^{\mathrm{V} 600 \mathrm{E}}$ cases and in melanoma with high p16, - 47\% of $B R A F^{\mathrm{V} 600 \mathrm{E}}$ cases, however, the difference was not statistically significant. There was a very significant difference between the frequency of BRAF ${ }^{\mathrm{V} 00 \mathrm{E}}$ samples in high p16 nevi $(85 \%)$ and high p16 melanoma (47\%) $(p<0.0001)$. We further subdivided samples according to the pathological classification and observed that in compound nevi, all samples showed a high p16 score (Figure $3 \mathrm{~F}$ ). In melanoma in situ and primary melanoma Clark III/IV, the frequency of $B R A F^{\mathrm{V} 600 \mathrm{E}}$ is higher in low p16 samples, however with no statistical significance. In contrast, metastatic melanoma samples with high p16 display a slightly higher frequency of BRAF ${ }^{\mathrm{V}}{ }^{00 \mathrm{E}}$ cases in comparison to the low p16 subgroup (60\% vs. $50 \%$ ) (Figure 3 F).

\section{Expression of Ki67}

Expression of Ki67 was evaluated in dysplastic nevi and melanoma cases. The number of dysplastic nevi cases expressing Ki67 in more than 10\% of cells was very low (Figure $4 \mathrm{~A}$ ). $80 \%$ of BRAF $\mathrm{F}^{\mathrm{V} 600 \mathrm{E}}$ and $83 \%$ of WT cases demonstrated a similar number of stained cells, which did not exceed $10 \%$ of Ki67 positive cells. In $44 \%$ of melanoma in situ with BRAF ${ }^{\mathrm{V} 600 \mathrm{E}}$ and in $70 \%$ of WT melano- ma samples, more than $10 \%$ of Ki67 positive cells were seen. All cases of primary melanoma Clark III/IV with BRAF ${ }^{\mathrm{V} 600 \mathrm{E}}$ and $66 \%$ of WT had more than $10 \%$ of $\mathrm{Ki} 67$ positive cells. In melanoma metastases with BRAF $F^{\mathrm{V} 600 \mathrm{E}}$ and WT, the fraction of cases with less than $10 \%$ of Ki67 positive cells was similar, approximately $20 \%$, however, there were $62 \%$ of $B R A F^{\mathrm{V} 600 \mathrm{E}}$ samples and $32 \%$ of WT cases with more than $50 \%$ of Ki67 positive cells (Figure 4 A). The average Ki67 IHC score is higher in melanoma (average $\mathrm{IHC}$ score $=5.225 \pm 3.092$ ) than in nevi (average IHC score $=2.364 \pm 0.809)(p$-value $=0.001)$, however it does not differ in pathological subgroups of melanoma, even when subdivided into BRAF ${ }^{\mathrm{V} 600 \mathrm{E}}$ and WT-expressing cases (Figures 4 B-D). However, when samples were divided into a low and a high Ki67 expressing group and the frequency of $B R A F^{\mathrm{V} 600 \mathrm{E}}$ mutation was tested in each pathological subgroup, we observed a higher frequency of BRAF $F^{\mathrm{V} 600 \mathrm{E}}$ in high Ki67 expressing subgroups of melanoma in situ (71\% vs. $42 \%$ in low Ki67 group), primary melanoma Clark III/IV (67\% vs. 50\% in low Ki67 group) and metastatic melanoma (63\% vs. $50 \%$ in low Ki67 group) (Figure $4 \mathrm{E}$ ).

\section{The correlation of p16 and Ki67 expression}

The correlation of p16 and Ki67 expression in melanoma samples was determined using Spearman rank correlation (Figure 5). We observed a statistically significant $(p<0.05)$ negative correlation between p16 IHC score and Ki67 IHC score in melanoma samples $(r=-0.3234)$. The presence of BRAF $F^{\mathrm{V} 600 \mathrm{E}}$ or WT does not affect the p16 and Ki67 correlation (data not shown). To present in the graph melanoma samples with identical p16 and Ki67 IHC scores, the color legend was utilized.

\section{Discussion}

In the last decade significant progress in genomic classification [2] and genetic evolution of skin melanoma from precursor lesions [3, 5, 6] has been achieved. Most of the current knowledge of melanoma is derived from the study of patients from populations of European descent [1]. However, there are no reports of BRAF $F^{\mathrm{V} 600 \mathrm{E}}$ driven evolution melanomas in the Polish population. Previous reports indicated CDKN2A variants associated with melanoma and other cancers in Poland [19-21, 25]. Moreover, our functional in vitro studies using vectors carrying 3'UTR sequence variants 500C $>\mathrm{G}$ and 540C $>$ T together with luciferase reporter gene transfected into multiple human melanoma cell lines demonstrated a very significant reduction (up to 4000 times) of the luminescence emitted by transfected cells [26]. This indicates that polymorphisms may modulate CDKN2A gene expression, thus the level of p16 in melanoma cells.

Beyond in vitro and mouse models $[7,8,27]$, there is only a single clinically focused report [28] on oncogenic BRAF ${ }^{6000}$ mutation and $p 16$ expression impact 
A

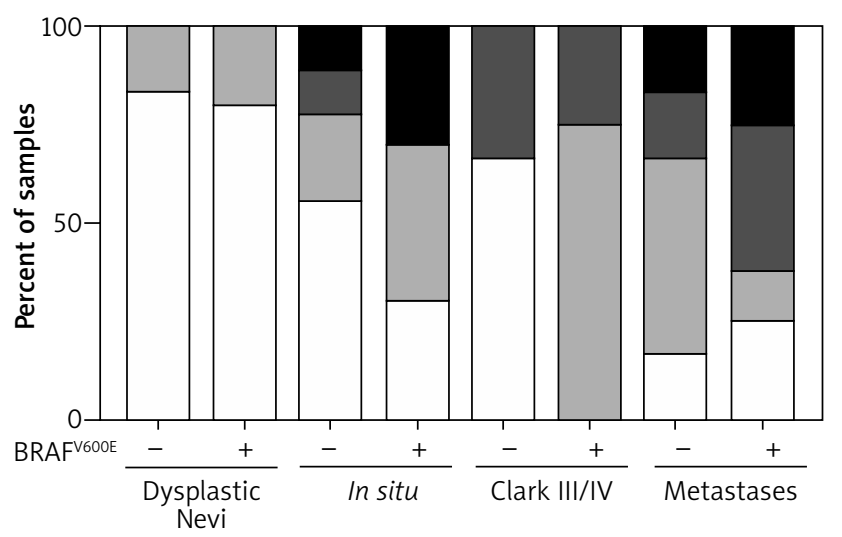

Percentage of p16-positive cells $\mathbf{n}$ 76-100\% $\square 51-75 \%$
B

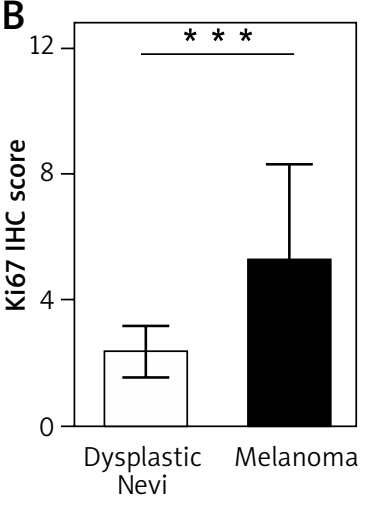

C

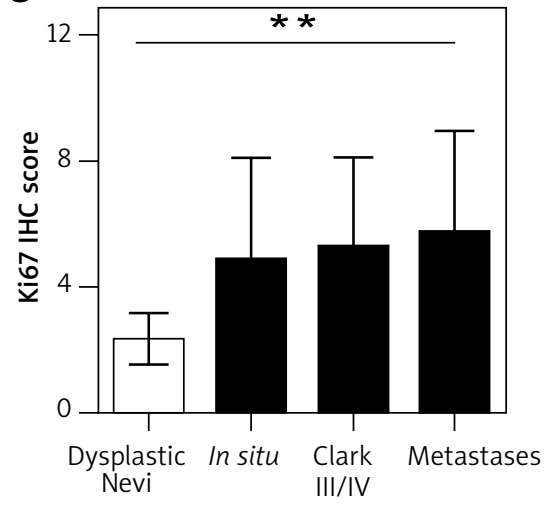

D

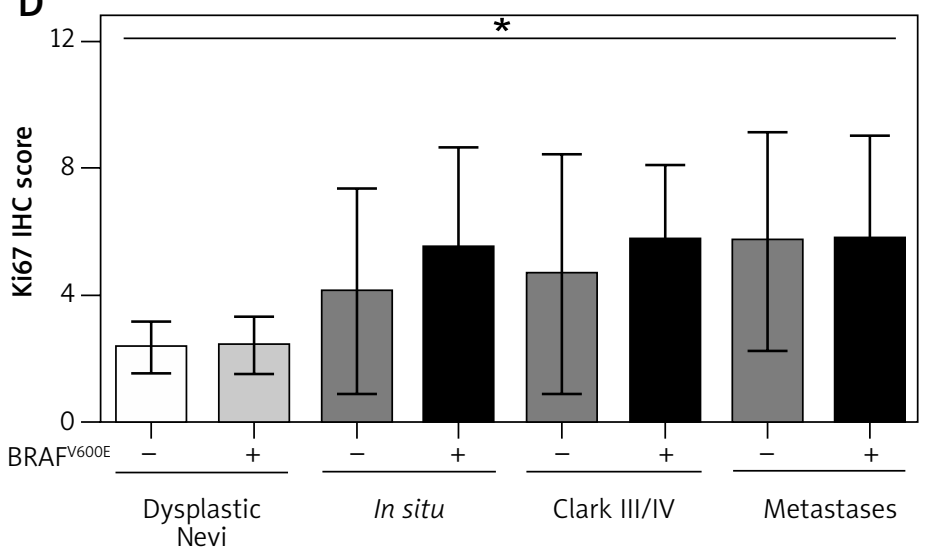

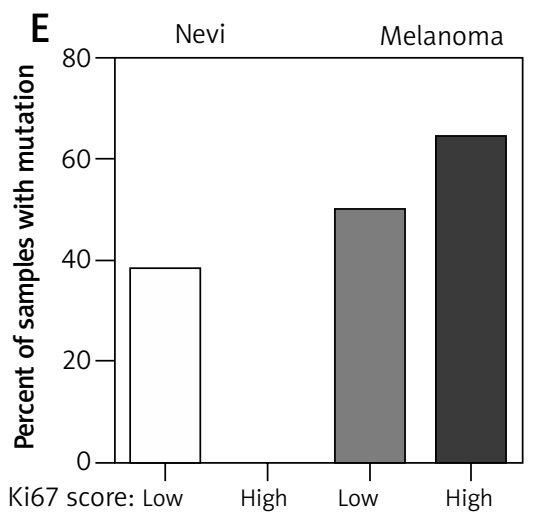

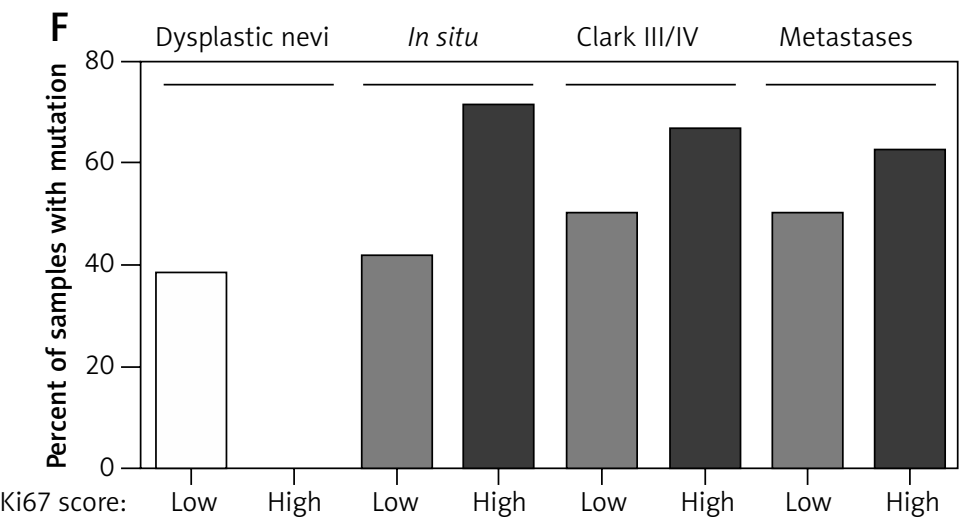

Figure 3. The Ki67 immunohistochemistry score differs significantly between dysplastic nevi and melanoma samples. A - The frequency of Ki67-positive cells in nevi and melanoma samples according to the pathological classification and the BRAF mutation status. 4-point percentage scale of the number of positive cells was used $(1=0-10 \%$ of positive cells, $2=11-50 \%, 3=51-75 \%$ and $4=76-100 \%)$. B - The average Ki67 IHC score is significantly higher in melanoma when compared to dysplastic nevi samples $(p=0.001)$. C - The average Ki67 IHC score in dysplastic nevi and melanoma according to the pathological classification. D - The average Ki67 IHC score in dysplastic nevi and melanoma samples according to the pathological classification and BRAF mutation differs significantly. $\mathrm{E}$ - The frequency of BRAF ${ }^{\mathrm{V} 600 \mathrm{E}}$ mutation in dysplastic nevi and melanoma samples according to the level of Ki67 IHC score (low score $\leq 6$, high score $>6$ ). F - The frequency of $\mathrm{BRAF}^{\mathrm{V} 600 \mathrm{E}}$ mutation in dysplastic nevi and melanoma samples according to the level of Ki67 IHC score (low score $\leq 6$, high score $>6)$ and the pathological classification. In dysplastic nevi there are no samples with high Ki67 IHC score. Error bars $=\mathrm{SD},{ }^{*} p<0.05,{ }^{* *} p<0.01,{ }^{* * *} p=0.001$ 


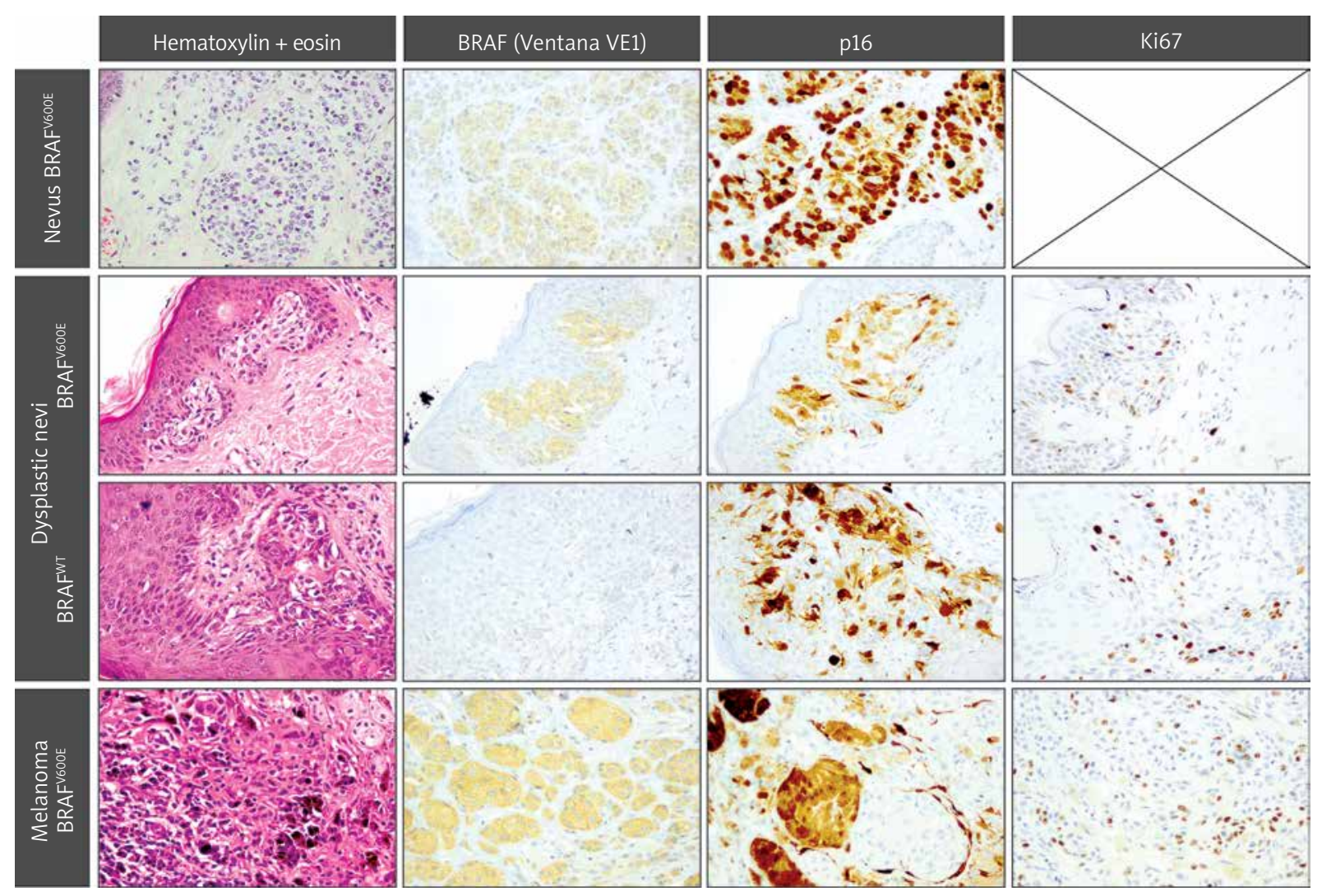

Figure 4. Representative images of BRAF (VE1), p16 and Ki67 immunohistochemical staining in nevi and melanoma samples. Dermal nevus with BRAF ${ }^{\mathrm{V}} 00 \mathrm{E}$ mutation, dysplastic nevi with BRAF ${ }^{\mathrm{G}}{ }^{\circ 0}$ and WT status and primary melanoma Clark III/IV with BRAF ${ }^{V} 600 \mathrm{E}$ mutation immunostained for specific markers are depicted

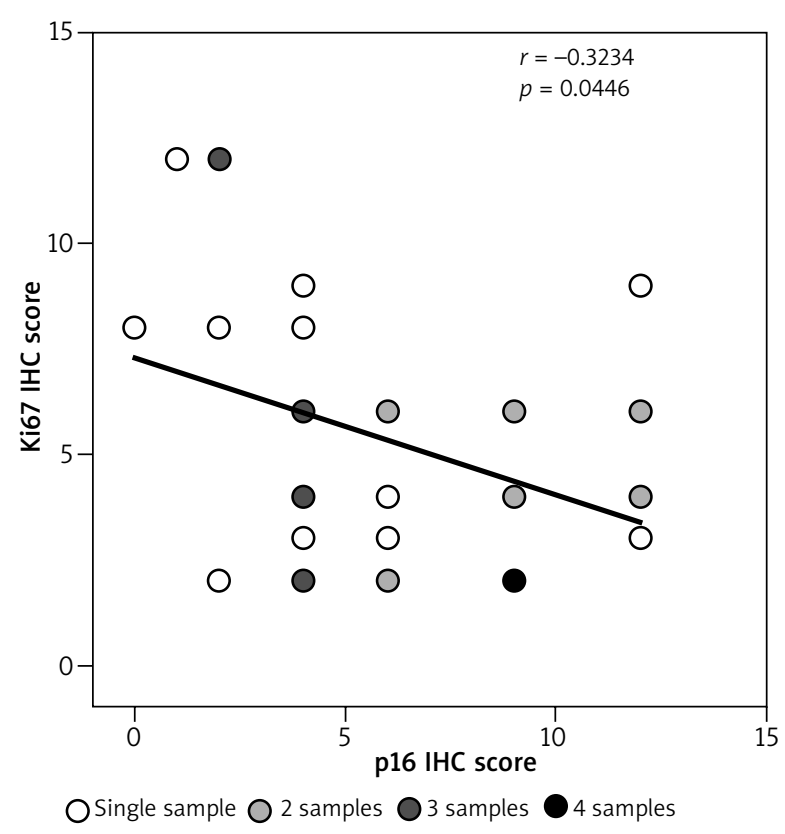

Figure 5. Spearman correlation of p16 and Ki67 IHC scores. The expression of p16 negatively correlates with the level of Ki67 in melanoma samples $(r=-0.3234 ; p=0.0446)$. Dots are colored as follows: white - single sample, light grey 2 samples, dark grey -3 samples, black -4 samples with specific p16 and Ki67 IHC score values on the growth rate of nevi and early melanoma in vivo. However, the growth rate in later phases of BRAF $F^{\mathrm{V} 00 \mathrm{E}}$ mutated melanoma development (metastases) in the context of p16 expression remains unexplored. Recently Tschandl et al. [29] has measured the growth rate of early melanoma and nevi in vivo using digital dermoscopy. They found that melanomas grew faster than nevi. Melanomas with $B R A F^{\mathrm{V} 600 \mathrm{E}}$ mutation grew significantly faster than WT. The effect of BRAF ${ }^{\mathrm{V} 600 \mathrm{E}}$ was not observed in melanocytic nevi. However, in their clinical material, frequency of $B R A F^{\mathrm{V} 600 \mathrm{E}}$ mutations was similar in melanoma and nevi. Moreover, they found no difference in p16 expression between nevi and melanoma however, they saw a higher growth rate of BRAF ${ }^{\mathrm{V} 600 \mathrm{E}}$ melanoma in vivo with negative $p 16$. In our studies we found a significant difference between $B R A F^{\mathrm{V} 600 \mathrm{E}}$ frequency in nevi and melanoma as well as p16 lower expression in BRAF $F^{\mathrm{V} 600 \mathrm{E}}$ mutated melanoma than nevi. Moreover, p16 expression level (score) negatively correlated with melanoma cells proliferation (Ki67). However, the final conclusion of Tschandl et al. [29] report related to negative correlation between p16 and in vivo growth rate of BRAF $F^{\mathrm{V} 600 \mathrm{E}}$ melanoma variant was identical with our conclusions. In addition, Tschandl et al. admitted a limitation of the inclusion of cases in their study, which a priori were preselected for digital monitoring. 
In our and Tschandl et al. [29] studies, only V600E $B R A F$ mutation was investigated, while there are other BRAF mutations such as G466E, S467L, G469R/E, V559E, $\mathrm{N} 581 \mathrm{H} / \mathrm{S} / \mathrm{T}$, and others [2], which we did not study. Moreover, in our WT group there are other driver mutated genes such as NRAS, which are found in nevi and melanoma and similarly as BRAF activate mitogen-activated protein kinase (MAPK) pathway and are regulated by p16 [29]. In our earlier study (data not published), we detected BRAF ${ }^{\mathrm{V} 600 \mathrm{E}}$ in $49 \%$ of metastatic melanoma, and various mutations of NRAS in $22 \%$ of the same cohort. Accordingly, the differences between BRAF ${ }^{\mathrm{V} 600 \mathrm{E}}$ and WT study groups as referred by us could be underestimated. In addition, Tschandl et al. [29], in melanoma associated nevi and uninvolved nevi, found BRAF ${ }^{\mathrm{V} 600 \mathrm{E}}$ mutation in $63 \%$ of melanomas, $65.2 \%$ of associated nevi and $50.0 \%$ of control nevi using the same VE1 anti-BRAF ${ }^{\mathrm{G} 00 \mathrm{E}}$ antibody for IHC. Significant differences in distribution of $B R A F$ and NRAS mutation were found. They observed the same difference between intensity of BRAF ${ }^{\mathrm{V} 00 \mathrm{E}} \mathrm{IHC}$ staining in nevi and melanoma as in our study. Based on the above results they challenged the current model of stepwise melanoma progression.

In cultured human melanocytes mutant BRAFV600 protein induced cells senescence by increasing expression of CDKN2A [15]. Accordingly, it has been hypothesized that to progress into melanoma, CDKN2A needs to be mutated or deleted. An experimental in vitro study demonstrated that simultaneous knockdown of $B R A F^{\mathrm{V} 600 \mathrm{E}}$ and CDKN2A in melanoma cells caused growth inhibition and apoptosis, while knockdown of BRAF $F^{\mathrm{V} 600 \mathrm{E}}$ or CDKN2A alone had no effect. Accordingly, it became indicative of a role of multiple tumor suppressors in the prevention of $B R A F^{\mathrm{V} 600 \mathrm{E}}$ oncogenesis [11]. Indeed, recently another CDK inhibitor, namely CDKN2B gene (p15) was found to be involved in nevi proliferation arrest in which $B R A F^{V 600 E}$ activation induced expression of TFG- $\beta$ signaling, which in turn up-regulated p15 and inhibited proliferation [8]. Taylor et al. [14] very recently reported on a random series of 29 nevi and 27 melanomas expression of p16 and p15. Both inhibitors demonstrated a high expression in nevi and substantially lower in melanoma on both protein and transcript levels. The differences were more significant for p15, which authors nominated for the biomarker candidate for distinguishing nevi from melanoma. However, they did not take into consideration $B R A F^{\mathrm{V} 600 \mathrm{E}} \mathrm{mu}$ tated melanomas and BRAF ${ }^{\mathrm{V} 600 \mathrm{E}}$ subtypes of melanocytic nevi or melanoma in various stages of development.

\section{Conclusions}

Finally, we found that $B R A F^{\mathrm{V} 600 \mathrm{E}}$ and $\mathrm{p} 16$ are more frequent in nevi than in melanoma in vivo. A significantly higher p16 expression was observed in mutated nevi than in WT, while in melanoma it was just the opposite. The proliferation rate of melanoma cells negatively cor- related with p16 expression. Our results confirm the hypothesis of the traditional model of stepwise tumor progression. However, further studies involving other driver mutations are required in order to fill the gap of our study, which is the better characterization of so called "WT" nevi and melanomas. Based on the obtained results and data available so far, just one study carried out in Austria [28], it is difficult to define the unique pattern of nevi-melanoma transition in the Polish population. Moreover, larger study groups are required.

\section{Acknowledgments}

The study was supported by PMU grant (502-0102233381-04678) and GPCC grant (18/2016 (133)).

\section{Conflict of interest}

The authors declare no conflict of interest.

\section{References}

1. Ossio R, Martin RR, Martinez-Said H, et al. Melanoma: a global perspective. Nature Rev Cancer 2017; 17: 393-4.

2. The Cancer Genome Atlas Network. Genomic classification of cutaneous melanoma. Cell 2015; 161: 1681-96.

3. Roh MR, Eliades P, Gupta S, Tsao H. Genetics of melanocytic nevi. Pigment Cell Melanoma Res 2015; 28: 661-72.

4. Pfeifer GP. Environmental exposures and mutational patterns of cancer genomes. Genome Med 2010; 2: 54.

5. Shain AH, Yeh I, Kovalyshyn I, et al. The genetic evolution of melanoma from precursor lesions. N Engl J Med 2015; 373: 1926-36.

6. Shain AH, Bastian BC. From melanocytes to melanomas. Nat Rev Cancer 2016; 16: 345-58.

7. Nasti TH, Cochran B, Tsuruta Y, et al. A murine model for the development of melanocytic nevi and their progression to melanoma. Mol Carcinogen 2016; 55: 646-58.

8. McNeal AS, Liu K, Nakhate V, et al. CDKN2B loss promotes progression from benign melanocytic nevus to melanoma. Cancer Discov 2015; 5: 1072-85.

9. Gill MJ, Celebi JT. B-RAF and melanocytic neoplasia. J Am Acad Dermatol 2005; 53: 108-14.

10. Pollock PM, Harper UL, Hansen KS, et al. High frequency of BRAF mutations in nevi. Nat Genet 2003; 33: 19-20.

11. Michaloglou C, Vredeveld LC, Soengas MS, et al. BRAF E600associated senescence-like cell cycle arrest of human nevi. Nature 2005; 436: 720-4.

12. Tsao H, Bevona C, Goggins W, et al. The transformation rate of moles (melanocytic nevi) into cutaneous melanoma: a population based estimate. Arch Dermatol 2003; 139: 282-8.

13. Tran SL, Haferkamp S, Scurr LL, et al. Absence of distinguishing senescence traits in human melanocytic nevi. J Invest Dermatol 2012; 132: 2226-34.

14. Taylor LA, O’Day C, Dentchev T, et al. p15 expression differentiates nevus from melanoma. Am J Pathol 2016; 186: 3094-99.

15. Conway C, Beswick S, Elliott F, et al. Deletion at chromosome arm $9 p$ in relation to BRAF/NRAS mutations and prognostic significance for primary melanoma. Genes Chromosomes Cancer 2010; 49: 425-38. 
16. Gao J, Aksoy BA, Dogrusoz U, et al. Interactive analysis of complex cancer genomics and clinical profiles using the cBioPortal. Sci Signal 2013; 6: 11.

17. Bertram CG, Gaut RM, Barrett EP, et al. An assessment of the CDKN2A variant Ala148Thr as a nevus/melanoma susceptibility allele. J Invest Dermatol 2002; 119: 961-5.

18. Bisio A, Nasti S, Jordan JJ, et al. Functional analysis of CDKN2A/ p16 INK4a 5'-UTR variants predisposing to melanoma. Hum Mol Genet 2010; 19: 1479-91.

19. Lamperska K, Karczewska A, Kwiatkowska E, Mackiewicz A. Analysis of mutations in the p16/CDKN2A gene in sporadic and familial melanoma in Polish population. Acta Bioch Po 2002; 49: 369-76.

20. Debniak T, Scott RJ, Huzarski T, et al. CDKN2A common variants and their association with melanoma risk: a population-based study. Cancer Res 2005; 65: 835-9.

21. Lamperska KM, Przybyla A, Kycler W, Mackiewicz A. The CDKN2a common variants: 148Ala/Thr and 500C/G in 3'UTR and their association with clinical course of melanoma. Acta Bioch Pol 2007; 54: 119-24.

22. Fu B, Wang Z, Li X, et al. Detection of BRAF V600E mutation in Langerhans cell histiocytosis using high-resolution melting analysis in decalcified, paraffin-embedded tissue. J Leuk 2013; 11: 1-8.

23. Bruno W, Martinuzzi C, Andreotti V, et al. Heterogeneity and frequency of BRAF mutations in primary melanoma: comparison between molecular methods and immunohistochemistry. Oncotarget 2017; 8: 8069-82.

24. Cerami E, Gao J, Dogrusoz U, et al. The cBio cancer genomics portal: an open platform for exploring multidimensional cancer genomics data. Cancer Discov 2012; 2: 401-4.

25. Debniak T, Gorski B, Huzarski T, et al. A common variant of CDKN2A (p16) predisposes to breast cancer. J Med Genet 2005; 42: 763-5.

26. Przybyła A, Lamperska K, Mackiewicz A. Analysis of sequence variants in the 3'UTR of CDKN2A gene in melanoma patients. Contemp Oncol 2015; 19: 276-9.

27. Conde-Perez A, Larue L. Human relevance of NRAS/BRAF mouse melanoma models. Eur J Cell Biol 2014; 93: 82-6.

28. Tschampl P, Berghoff AS, Preusser M, et al. Impact of oncogenic BRAF mutations and p16 expression on the growth rate of early melanomas and naevi in vivo. $\mathrm{Br} J$ Dermatol 2016; 174: 364-70.

29. Tschandl P, Berghoff AS, Preusser M, et al. NRAS and BRAF mutations in melanoma-associated nevi and uninvolved nevi. PLoS One 2013; 8: e69639. 